ORIGINAL ARTICLES

\title{
Interleukin-6: A Sensitive Parameter for the Early Detection of Neonatal Sepsis
}

\author{
M Khaled Noor ${ }^{1}$, M Shahidullah ${ }^{2}$, Hamidur Rahman $^{3}$, Mahbub Mutanabbi $^{4}$ \\ ${ }^{1}$ Associate Prof. Neonatology, BSMMU, ${ }^{2}$ Professor, Neonatology, BSMMU, ${ }^{3}$ Professor of Paediatrics, BSMMU, ${ }^{4}$ Assistant Professor Peadiatrics, \\ BSMMU, Dhaka.
}

\begin{abstract}
:
Background: Neonatal sepsis is a major cause of neonatal mortality and morbidity throughout the world. Though blood culture is the gold standard and has higher sensitivity and specificity over the hematological value and cytokine, it is not available in our community health situation and also in most of health care facilities. It is also time consuming. Therefore hematological value and interleukin- 6 can be evaluated for the early diagnosis of neonatal bacterial infection. Objective: This study was conducted to see the usefulness of IL-6 as an early marker of neonatal sepsis and also to compare the sensitivity in comparison with CRP, hematological value and blood culture. Study Design: It was a quasy experimental study. Setting: This study was carried out in the neonatal unit of pediatric department, BSMMU during the period of September, 2005 to February, 2006. Method: Forty five suspected septic cases were enrolled in the study and thirty healthy newborn were taken for comparison. Venous blood sample from peripheral vein was collected on the $1^{\text {st }}$ day of symptoms and/or $1^{\text {st }}$ day of admission and was sent for IL-6 estimation within half an hour and estimation of IL-6 was done by using immunolyte DPC USA which employed automated chemiluminescent immunoassays. Results: Out of forty five cases of suspected-neonatal sepsis, IL-6 were positive in twenty five cases. In culture proven sepsis $100 \%$ cases had raised IL-6. In control group only five babies had raised IL-6. Three cases were culture positive, of which all were also positive for IL-6 (100\%). Among the cases twenty six were CRP positive, of which twenty were also positive for IL-6 (76.92\%). Conclusion: In the present study IL-6 was found to be an early marker of neonatal infection. Sensitivity was more than CRP and other hematological parameter in the first twenty four hours.
\end{abstract}

Key word: Interleukin-6, Neonatal Sepsis.

[BSMMU J 2008; 1(1): 1-5]

\section{Introduction:}

Neonatal infections are a frequent and important cause of neonatal morbidity and mortality. Neonatal septicemia is a clinical syndrome of systemic illness accompanied by bacteraemia occurring in the $1^{\text {st }}$ day of life $e^{1}$. Neonatal sepsis may be categorized as early onset and late onset sepsis. Eighty five percent of newborns with early onset infection present with in 24 hours, five percent present at 24-48 hours and smaller percentage of patients between 48 hours and 6 days of life.

In the global perspective, the microorganisms most commonly associated with early onset infection include group B streptococcus (GBS), Escherichia Coli, Heamophilus influenzae and Listeria monocytogens ${ }^{2}$. In case of late onset infection causative organisms are coagulase negative staphylococci, staphylococcus arueus, E. coli, Klebsiella, Pseudomonas, Enterobacter, Candida, GBS, Serratia, Acinobacter and anaerobes ${ }^{3}$.

Address for correspondence: Dr. M Khaled Noor, Associate Prof. (Neonatology) Room \# 209, Block - C, Bangabandhu Sheikh Mujib Medical University (BSMMU), Dhaka, Bangladesh.
The reported incidence of neonatal sepsis varies from $7.1^{4}$ to $38{ }^{5}$ per 1000 live births in Asia, from $6.5^{6}$ to $8.9^{7}$ per 1000 live births in Africa, and from $3.5^{8}$ to $8.9^{9}$ per 1000 live births in South America and the Caribbean. By comparison, rates reported in the United States and Australasia range from 1.5 to 3.5 per 1000 for early onset sepsis and up to 6 per 1000 live births for let onset sepsis, a total of 6-9 per 1000 for neonatal sepsis. ${ }^{10-13}$.

CRP (C reactive protein), hematological parameter and blood culture have good predictive value in the detection of neonatal sepsis ${ }^{14} 15$. CRP is an acute phase reactant, which has been used in the diagnosis of bacterial infection in the neonates ${ }^{16}$. IL-6 is a proinflammatory cytokine produced by monocytes and macrophages activated by bacterial infection. ${ }^{17}$ Interleukin- 6 is one of the markers of infection which appears earlier and remains elevated in first 24 hours after which it declines. This initiates the formation of CRP which is elevated 24-48 hours after the onset of infection and persist upto the time until the infection is not resolved. As interleukine-6 plays a critical role in the induction of CRP synthesis in the liver it was 
hypothesized that this cytokine could be detected earlier in the blood than CRP during the course of bacterial infection. IL-6 decreased in 24 hours to undetectable level in the majority of the infected infants ${ }^{18}$. Sensitivity of IL6 in CRP negative newborns on admission was 100\% in newborns with blood culture positive and clinical sepsis 18. IL-6 was more sensitive than CRP in infected newborn on admission $73 \%$ vs $58 \%{ }^{18}$.

Though blood culture is the gold standard and has higher sensitivity and specificity over the hematological value and cytokine but this highly sensitive microbiological parameter is not available in our community health situation and it is time consuming also. Therefore hematological value and interleukin- 6 can be evaluated for the early diagnosis of neonatal bacterial infection. This study was carried out to measure the level of interleukin6 for the diagnosis of neonatal sepsis and compare the level of interleukin-6 with CRP, hematological value and blood culture.

\section{Methodology}

Study design:

It was a quasy experimental study. This study was carried out in the neonatal unit, Department of Pediatric, BSMMU during the period of September 2005 to February 2006. Forty five cases of suspected septicemia were enrolled in the study and thirty healthy newborn were taken for comparison. The samples were randomly collected from neonatology unit, Department of Pediatrics BSMMU. In this hospital, high risk neonates of suspected sepsis are referred from obstetric department of the same hospital (in born) as well as from different hospitals and clinics of Dhaka and outside Dhaka (out born).

\section{Inclusion Criteria:}

i. Newborn babies who had more than one clinical features of suspected sepsis.

ii. In absence of clinical features the babies having risk factors (one major or two minor) were also taken as suspected septic cases. ${ }^{19}$

\section{Risk factors:}

Major risk factors:

Rupture of membrane $>24 \mathrm{hrs}$., Chorioamniontis Maternal intrapartum fever $>100.4^{0} / 38^{0} \mathrm{C}$, sustained fetal heart rate $>160$.

\section{Minor risk factors:}

Rupture of membrane > 12hrs., Maternal WBC count > 15000/cmm, Low APGAR score at 5 min. Preterm labour, Low birth weight. Foul Lochia, maternal colonization
Exclusion Criteria: Neonates with major congenital abnormality

Measurements of outcome variables:

1. Clinical features: Lethargy, Reluctant to feed etc.

2. Hematological variables: Total count of WBC \& Platelet count.

3. Immunological variables: CRP, IL- 6

\section{Procedure and Technique:}

After taking a careful history thorough physical examination was carried out. A specified questionnaire was designed and attendants were interviewed. The answer were compiled by the investigator.

\section{The following investigations had been done:}

In the $1^{\text {st }}$ day of symptom and $1^{\text {st }}$ day of admission, CBC (total leukocyte count, platelet count), blood for culture and sensitivity (C/S), C-reactive protein (CRP) and blood for interleukin-6 was done and after 48-72 hours of symptom blood for C-reactive protein (CRP) was done. On evaluation of various Haematological parameters, TLC $<5 \times 10^{9}$ / L ( leukopenia) or $25 \times 10^{9} / \mathrm{L}$ (leukocytosis), Thrombocytopenia if platelet count $<100 \times 10^{9}$ / L was taken as standard. For blood culture in thirty cases blood was taken in conventional bottle and fifteen cases blood was taken in FAN bottle.Cytokines (IL-6) were measured using IMMULITE, DPC, USA, which employed automated chemiluminescent

immuno- assays. As per instruction in the manual provided by the company normal value of IL- 6 is $<10 \mathrm{pg} / \mathrm{ml}$.CRP value was estimated by latex agglutination method with CRP kit manufactured by CHRONOLAB Ag, Switzerland. As per instruction in the manual provided by the company CRP value $<10 \mathrm{mg} / \mathrm{L}$ was taken as negative. Data was compiled and statistically analyzed by using SPSS version 10 .

\section{Results}

Table-I Shows that Lethargy (37.8\%), reluctant to feed (33.3\%), abdominal distension (33.3\%), respiratory distress (31.1\%), apnoea (22.2\%) and hyperthermia $(22.2 \%)$ were the common features of neonatal sepsis in this series.

In this study, suspected septic babies had high leukocyte count (>25000). This count was compared with IL-6 level. IL-6 had a high sensitivity (85.71\%), negative predictive value (95\%) with an accuracy of 56\% (Table -II)

Table-III shows the comparison of decreased platelet count (<100000) with IL-6 level in suspected sepsis cases. IL-6 had high sensitivity (88\%), specificity (51.35\%) with an accuracy of $56 \%$. 
IL-6 level was compared with CRP positive suspected sepsis cases (Table-IV). In twenty six CRP positive cases, IL-6 was positive in twenty and among nineteen CRP negative cases IL-6 was positive in five. Here sensitivity (77\%), specificity (74\%), positive predictive value (80\%) and negative predictive value (70\%) were high with an accuracy of $78 \%$.

In comparison of IL-6 with blood culture (Table -V) three babies had culture proven sepsis and forty two culture negative sepsis. Culture proven three babies were also IL6 positive and among culture negative forty two babies, twenty two were IL-6 positive. Sensitivity of IL-6 was (100\%), and negative predictive value was also found (100\%) with an accuracy of 51.11\%.

When control and case groups were compared for CRP and IL-6, the level of significance was much higher for IL-6 (<.001) than CRP $(<.05)$ (Table-VI).

Table-I

Presenting features of the cases $(n=45)$

\begin{tabular}{lcc}
\hline Presenting features & Number of patients & Percentage \\
\hline Lethargy & 17 & 37.8 \\
Reluctant to feed/vomiting & 15 & 33.3 \\
Abdominal distension & 15 & 33.3 \\
Respiratory distress & 14 & 31.1 \\
Apnoea & 10 & 22.2 \\
Hyperthermia & 10 & 22.2 \\
Bleeding & 6 & 13.3 \\
Hypothermia & 4 & 8.8 \\
Sclerema & 2 & 4.4 \\
\hline
\end{tabular}

Table II

Validity of IL 6 test in comparison to total leukocyte count in cases $(n=45)$

\begin{tabular}{lcccccc}
\hline \multirow{2}{*}{$\begin{array}{l}\text { Total leukocyte } \\
\text { count }\end{array}$} & \multicolumn{4}{c}{ IL 6 test } \\
\cline { 3 - 6 } & No. & No. & $(\%)$ & & No. & $(\%)$ \\
\cline { 3 - 6 } \cline { 5 - 7 } $\begin{array}{l}\text { Raised } \\
(>25000)\end{array}$ & 7 & 6 & $(85.7)$ & & 1 & $(14.3)$ \\
Normal & 38 & 19 & $(50.0)$ & & 19 & $(50.0)$ \\
\hline Total & 45 & 25 & $(55.6)$ & & 20 & $(44.4)$ \\
\hline
\end{tabular}

\begin{tabular}{lll}
\hline Sensitivity & $:$ & $85.71 \%$ \\
Specificity & $:$ & $50 \%$ \\
Positive predictive value & $:$ & $24 \%$ \\
Negative predictive value & $:$ & $95 \%$ \\
Accuracy & $:$ & $55.56 \%$
\end{tabular}

Table III

Validity of IL 6 test in comparison to platelet count in cases $(n=45)$

\begin{tabular}{lcccccc}
\hline & & \multicolumn{4}{c}{ IL 6 test } \\
\cline { 3 - 4 } Platelet count & & \multicolumn{2}{c}{ Positive } & & \multicolumn{2}{c}{ Negative } \\
\cline { 3 - 4 } \cline { 6 - 7 } & No. & No. & $(\%)$ & & No. & $(\%)$ \\
\hline Decreased & 8 & 7 & $(87.5)$ & & 1 & $(12.5)$ \\
$(<100000)$ & & & & & & \\
Normal & 37 & 18 & $(48.6)$ & & 19 & $(51.4)$ \\
\hline Total & 45 & 25 & $(55.6)$ & & 20 & $(44.4)$ \\
\hline Sensitivity & & & $:$ & & $87.5 \%$ \\
Specificity & & & $:$ & & $51.35 \%$ \\
Positive predictive value & $:$ & & $28 \%$ \\
Negative predictive value & $:$ & & $95 \%$ \\
Accuracy & & & $:$ & & $57.78 \%$
\end{tabular}

Table IV

Validity of IL 6 test in comparison to CRP in cases. $(n=45)$

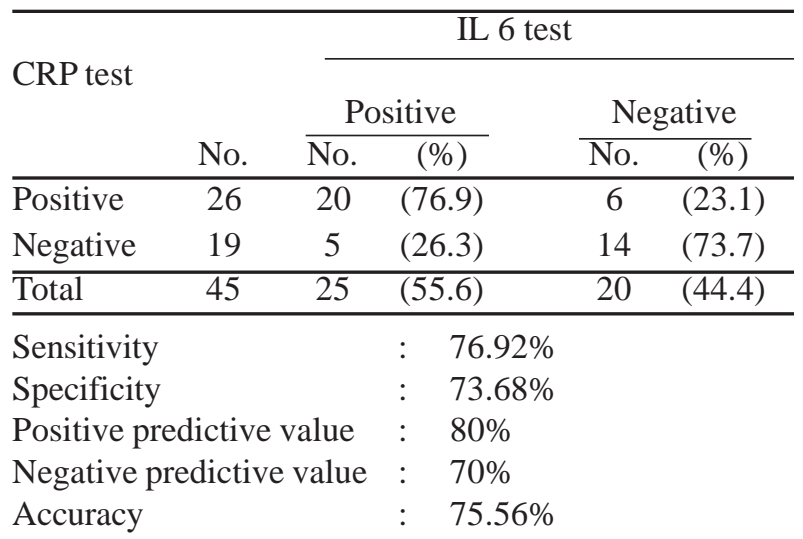

Table V

Validity of IL 6 test in comparison to blood culture in cases. $(n=45)$

\begin{tabular}{lcccccc}
\hline \multirow{2}{*}{ Blood culture } & \multicolumn{4}{c}{ IL 6 test } \\
\cline { 2 - 4 } & & \multicolumn{2}{c}{ Positive } & & \multicolumn{2}{r}{ Negative } \\
\cline { 2 - 4 } \cline { 6 - 7 } & No. & No. & $(\%)$ & & No. & $(\%)$ \\
\hline Positive & 3 & 3 & $(100.0)$ & & 0 & \\
Negative & 42 & 22 & $(52.4)$ & & 20 & $(47.6)$ \\
\hline Total & 45 & 25 & $(55.6)$ & & 20 & $(44.4)$ \\
\hline Sensitivity & & $:$ & $100 \%$ & \\
Specificity & & $:$ & $47.61 \%$ & \\
Positive predictive value & $:$ & $12 \%$ & \\
Negative predictive value & $:$ & $100 \%$ & \\
Accuracy & & $:$ & $51.11 \%$ &
\end{tabular}


Table VI

Comparison of CRP and IL 6 levels between cases $(n=45)$ and control $(n=30)$

\begin{tabular}{|c|c|c|c|c|c|}
\hline \multirow[t]{2}{*}{$\begin{array}{l}\text { Neonatal } \\
\text { sepsis }\end{array}$} & \multicolumn{2}{|c|}{$\begin{array}{c}\text { Control } \\
(\mathrm{n}=30)\end{array}$} & \multicolumn{2}{|c|}{$\begin{array}{l}\text { Case } \\
\text { (n 45) }\end{array}$} & \multirow[t]{2}{*}{$P$ value } \\
\hline & No. & (\%) & No. & (\%) & \\
\hline \multicolumn{6}{|l|}{ CRP } \\
\hline \multirow[t]{2}{*}{ Positive } & 9 & $(30.0)$ & 26 & (57.8) & \\
\hline & & & & & $<0.05^{*}$ \\
\hline Negative & 21 & $(70.0)$ & 19 & $(42.2)$ & \\
\hline \multicolumn{6}{|l|}{ IL 6} \\
\hline \multirow[t]{2}{*}{ Positive } & 5 & (16.7) & 25 & (55.6) & \\
\hline & & & & & $0.001^{* * *}$ \\
\hline Negative & 25 & (83.3) & 20 & $(44.4)$ & \\
\hline
\end{tabular}

Chi square test

$* / * * *$ = Significant

Sensitivity of IL-6 in neonatal sepsis : $55.6 \%$

Specificity of IL-6 in neonatal sepsis : $83.3 \%$

\section{Discussion}

In neonatal sepsis commonly observed clinical features were lethargy (37.8\%), reluctant to feed (33.3\%), abdominal distension (33.3\%), respiratory distress (31.1\%), apnoea (22.2\%) and hyperthermia (22.2\%). A good number of babies had more than one clinical features. Theses observations are similar to the findings of other workers $^{20-22}$. Out of 45 cases of suspected neonatal sepsis blood culture was found positive in only 3 cases and negative in 42 cases. The validity of IL- 6 test in comparison to blood culture cases sensitivity was $100 \%$, specificity $47.61 \%$, positive predictive value $12 \%$, negative predictive value $100 \%$ and test of accuracy $51.11 \%$. In many studies the incidence of culture positive sepsis was not more than $10 \%{ }^{23}$. In our study less number of culture positive reports may be due to late arrival and sample collection after giving antibiotic. This conforms with the study of others ${ }^{24}$. Marked abnormality of leukocyte count was observed in only $15.5 \%$ cases, but in 84.5\% patient's leukocyte count was within the normal limit. The possibility of septicemia cannot be ruled out by normal leukocyte count. The leukocyte was not increased in all cases probably due to early collection, lab errors in counting and previous low levels. The validity of IL-6 in comparison to the total leukocyte count for cases was identified i.e. sensitivity $95.71 \%$, specificity $50 \%$, positive predictive value $24 \%$, negative predictive value $95 \%$, accuracy $55.56 \%$.

Out of 45 cases of suspected sepsis thrombocytopenia was observed in $17.8 \%$ and normal count $82.2 \%$. The validity IL-6 test in comparison to platelet count for cases was identified i.e. sensitivity $87.5 \%$, specificity $51.4 \%$, positive predictive value $28 \%$, negative predictive value $95 \%$, accuracy of $57.8 \%$. Out of 45 cases of suspected neonatal sepsis, CRP was raised in $57.8 \%$ and negative in $42.2 \%$. In culture proven sepsis, $100 \%$ cases had raised CRP which is an important marker of sepsis. Similar findings were reported by other investigators $20,24,25$. In control group 9 babies (30\%) had raised CRP. One baby had history of PROM, 5 babies had history of mild perinatal asphyxia and 3 babies had meconium aspiration syndrome (MAS), which may be associated with raised CRP 26,27 . Value of CRP compared between cases and controls was found statistically significant. Validity of IL-6 test in comparison to CPR in cases was identified i.e. sensitivity $76.92 \%$, specificity $74.68 \%$, positive predictive value $80 \%$, negative predictive value $70 \%$, accuracy $75.92 \%$. This is consistent with the finding of Magudumana $2000^{24}$. In this study in cases of suspected neonatal sepsis the value of IL-6 was raised in $55.6 \%$ cases and was normal in $44.4 \%$. This relatively high figure of normal IL-6 in cases was due to delay in bringing the patient in hospital. It is known that IL-6 is an early marker and the raised level start declining usually after 24 hours. In control group IL6 was found positive in 5 babies (16.7\%) and negative in 25 babies (83.3\%). The validity of IL-6 for cases was identified i.e. sensitivity $55.6 \%$ and specificity $83.3 \%$. The levels of IL-6 in comparison to control and cases is statistically significant ( $\mathrm{p}$ value is $<.001$ ).

\section{Conclusion}

Interleukin-6 is a very early marker in the diagnosis of neonatal infection. In the present study IL-6 level was raised in suspected septic cases. IL-6 was positive in more cases within twenty four hours of the onset of sepsis in comparison to other tests. It may be concluded that estimation of IL-6 done at the time of onset of signs and symptoms suggestive of infection is useful in the early diagnosis of neonatal sepsis.

\section{References}

1. Barbara JS. Infection of the neonatal infant. In: Behrman RE, Kliegman RM, Jenson HB, Stanton BF. editors. Nelson textbook of pediatrics. $18^{\text {th }}$ ed. Philadelphia: WB Saunders Company; 2008. p. 794-811.

2. Placzek MM, Whitclow A. Early and late neonatal septicemia. Arch Dis Child 1983; 58: 728-731. 
3. Stoll BJ, Gordon T, Korones SB. Early onset sepsis in very low birth weight neonates: a report from the National Institute of Child Health and Human Development Neonatal Research Network. J Pediatrics 1996; 129: 72-80.

4. Lim NL, Wong YH, Boo NY. Bacteremic infections in neonatal intensive care unit: a nine months survey. Med J Malaysia 1995; 50: 59-63,

5. Tallur SS, Kasturi AV, Nadgir SD. Clinico-bacteriological study of neonatal septicemia in Hubli. Indian J Pedialr 2000; 67: 16974

6. Airede Al. Neonatal septicaemia in an African city of high altitude. Trop Pediatr 1992; 38: 189-91.

7. The WHO multicentre study group. Clonical prediction of serious bacterial infections in young infants in developing countries, Pediatr Infect Dis J 1999; 18: 23 - 31.

8. Moreno MT, Varas S, Poveda R, Saezllorens X. Neonatal sepsis meningitis in a developing Latin American Country. Pediatr Infect Dis 1994;13: $516-520$.

9. Robilland PY, Nabeth P, Hulsey TC, Sergent MP, Perinania J, Janky E. Neonatal bacterial septicemia in a tropical area: fouryear experience in Guadelupe. Acta Paediatr 1993; 82: 678-679.

10. Hyde TB, Hilger TM, Reingold A. Trends in incidence and antimicrobial resistance of early onset sepsis: population -based surveillance in San Francisco and Toronto. Pediatrics 2002; 110: 690-5.

11. Scuchat A, Zywicki SS, Dinsmoor MJ. Risk factors and opportunities for prevention of early-onset neonatal sepsis: a multicenter case- control study. Pediatrics 2000; 105: 21-6.

12. Heath PT, Yussoff NK, Baker O. Neonatal meningitis. Arch Dis Child Fetal Neonatal ed 2003; 88: 173-8.

13. Isaacs D, Royle JA. Intrapartum antibiotics and early-onset neonatal sepsis caused by group B Streptococcus and by other organisms in Australia. Pediatr Infect Dis J 1999; 18: 524-8.

14. Manucha V, Rusia U, Sikka M, Faridi MMA and Madan N. Utility of haematological parameters and C-reactive protein in the detection of neonatal sepsis. J. Paediatrics and Child Health 2002; 38: 459-464.
15. Nuntnarumit P, Pinkaew O, Kitiwanwanich S. Predictive values of serial C-reactive protein in neonatal sepsis. J Med Assoc Thai 2002; 85; 1151-1158.

16. Mathur NB. Neonatal sepsis. Indian Pediatr 1996; 33: 663-670.

17. De Bont ESJM, Martens A, Van Raan J, Samson G, Fetter WPF, Okken A, etal. Diagnostic value of plasma levels of tumor necrosis factor (TNF- $\alpha$ ) and interleukin-6 in new borns with sepsis. Acta Pediatr 1994; 83: 696-9.

18. Buck C, Bunodschu J, Gallati H, Bartmann P, Pohlawadt F. Interleukin-6: a sensitive parameter for the early diagnosis of neonatal bacterial infection. Pediatrics 1994; 93: 54-8.

19. Guerina NG. Bacterial and Fungal infection. In: Cloherty JP, Stark AR, editors. Manual of Neonatal Care. $4^{\text {th }}$ ed. Philadelphia: Lippincott-Raven Publishers 1998; 271-300.

20. Mannan MA. Utility of C-reactive protein (CRP) and Hematological parameters in the detection of neonatal sepsis. Thesis for MD Neonatology Examination BSMMU, 2004.

21. Daoud AS, Abuekteish F, Obeidat A, El-Nassir Z, Al-Rimawi H. The changing face of neonatal septicemia. Ann Trop Paediatr 1995; 15: 93-96.

22. Gupta P, Murali MV, Faridi MMA, Kaul PB, Ramchandran VG, Talwar V. Clinical profile of Klebsiella septicemia in neonates. Indian J Pediatr 1993; 60: 565-572.

23. Hossain MM. Neonatal sepsis: report from different Institutes. Proceedings of the First BNF CME Seminar on Neonatal sepsis; October 21, 2003. Dhaka, Bangladesh Neonatal Forum, 2003.

24. Magudumana MD, Ballot DE, Copper PA, Cory BJ, Vi 1 Jzoen E, Carter AC. Serial IL-6 measurements in the early diagnosis of neonatal sepsis. J Trop Pediatr 2000; 46: 267-277.

25. Polin RA, Geme JWS. Neonatal sepsis. Adv Paediatr Infect Dis 1992; 7: 25-60.

26. Gardes JS, Polin R. Early diagnosis and treatment of neonatal sepsis.Indian J Pediatr 1998; 65: 63-78.

27. Kawamura M, Nishida H. The usefulness of serial C-reactive protein measurement in managing neonatal infection. Act Paediatr 1995; 84:10-13. 